\title{
Complete response to pyrotinib combined with letrozole as first- line treatment of HER2-positive/HR-positive breast cancer: a case report
}

\author{
Ning Xie, Liping Liu, Can Tian, Zheyu Hu, Quchang Ouyang \\ Department of Breast Cancer Medical Oncology, Hunan Cancer Hospital, the Affiliated Cancer Hospital of Xiangya Medical School, Central South \\ University, Changsha, China \\ Correspondence to: Quchang Ouyang. Department of Breast Cancer Medical Oncology, Hunan Cancer Hospital, the Affiliated Cancer Hospital of \\ Xiangya Medical School, Central South University, 283 Tongzipo Road, Changsha 410013, China. Email: ouyangquchang@hnca.org.cn.
}

\begin{abstract}
Approximately $15-20 \%$ of breast cancer patients are epidermal growth factor receptor 2 (HER2)-positive, and about half of these are also hormone receptor (HR)-positive. The mainstay treatment for HER2-positive/HR-positive patients is anti-HER2 treatment combined with chemotherapy. However, many patients are not suitable for this treatment regimen due to their poor physical health and inability to tolerate chemotherapy. Pyrotinib is a novel, irreversible tyrosine kinase inhibitor (TKI) with activity against EGFR/HER1, HER2, and HER4. Several studies have shown pyrotinib's anti-tumor activity and safety profile in treating HER-2 positive breast cancer patients, but its effect on metastatic breast cancer (MBC) when combined with letrozole as a first-line treatment remains to be verified. Here, we present a case of a 50-year-old postmenopausal HER2-positive/HR-positive breast cancer patient who received pyrotinib plus letrozole as a first-line treatment following a diagnosis of left axillary lymph node and double lung metastases after modified radical mastectomy for left breast cancer. Two months after administration of combined pyrotinib and letrozole, a complete response (CR) was confirmed by CT scan. The patient experienced only mild and tolerable adverse events. At the time of writing, the patient was still alive without any recurrence. Our case indicates that the combined therapy of pyrotinib plus letrozole may/can be a promising treatment option for patients with HER2-positive/HR-positive MBC. Nevertheless, further evidence is needed to verify this conclusion.
\end{abstract}

Keywords: HER2+/HR+ breast cancer; anti-HER2 targeted therapy; pyrotinib; metastatic breast cancer (MBC); case report

Submitted May 25, 2021. Accepted for publication Aug 16, 2021.

doi: 10.21037/atm-21-3978

View this article at: https://dx.doi.org/10.21037/atm-21-3978

\section{Introduction}

Globally, breast cancer is the most common malignant tumor in women and poses a serious threat to women's life and health (1). About $20 \%$ of breast cancers are epidermal growth factor receptor 2 (HER2)-positive, which are aggressive and have a poor prognosis (2). HER2 is also an important target in breast cancer treatment. Among HER2-positive breast cancer patients, approximately half are endocrine-dependent, and postmenopausal patients with an HR-positive status are eligible to receive aromatase inhibitor therapy (3). National Comprehensive Cancer Network (NCCN) guidelines recommend that HER2+/ $\mathrm{HR}+$ breast cancer patients receive anti-HER2 therapy combined with chemotherapy, and some patients who are not suitable for chemotherapy can use anti-HER2 therapy combined with endocrine therapy (4). Pyrotinib is a novel, irreversible HER2 tyrosine kinase inhibitor (TKI) which have gained approval for treating HER2-positive advanced or metastatic breast cancer in China (5). Letrozole is a nonsteroidal, third-generation aromatase inhibitor which 
have shown anti-tumor activity in postmenopausal women with HR-positive breast cancer (6). Up to now, only study protocol of pyrotinib combined with letrozole in treating HER 2+/HR + breast cancer has been published, and the effect and safety profile of this combination regimen have not been demonstrated yet (7). Herein we present a case of a HER2+/HR+ breast cancer patient with left axillary lymph node and double lung metastases after left breast modified radical mastectomy, who achieved complete response (CR) after receiving pyrotinib combined with letrozole as a first-line treatment. We present the following article in accordance with the CARE reporting checklist (available at https://dx.doi.org/10.21037/atm-21-3978).

\section{Case presentation}

In February 2015, a 45-year-old woman accidentally found a painless lump in her left breast and was subsequently diagnosed with breast cancer at her local hospital. She then underwent enlarged resection of the left breast mass and a modified radical mastectomy for left breast cancer. The postoperative pathological report described left breast invasive ductal carcinoma, Grade II; ER (3+, 60\%), PR (3+, 70\%), HER2 (3+), and Ki-67 (50\%) with no nodal involvement. A preliminary comprehensive consultation recommended the patient receive chemotherapy combined with anti-HER2 therapy, endocrine therapy, and regular review. The patient started adjuvant therapy using docetaxel, carboplatin, and trastuzumab (TCbH) for 6 cycles followed by toremifene oral treatment, which lasted 4 years from May 5, 2016 to August 13, 2020. During this period, the patient was reviewed regularly and her condition was stable.

On August 13, 2020, the patient was re-examined at Hunan Cancer Hospital and was diagnosed with disease progression with metastases in the left axillary lymph node and both lungs. Color Doppler ultrasound showed hypoechoic nodules in the left upper chest-wall and left anterior axillary, and thyroid nodules consistent with TIRADS3 type, benign disease. A contrast-enhanced chest computed tomography (CT) scan revealed enlarged left axillary lymph nodes and multiple small nodules in the lungs, consistent with MBC. The results of the examination showed that the patient's condition was worsening. The patient underwent a complete CT re-examination on August 26, 2020, which showed similar results to the initial scan (Figure 1A,1B). A cardiac color Doppler ultrasound indicated tachycardia, decreased diastolic function of the left heart, but normal systolic function.

After consultation with selective experts, trastuzumab + chemotherapy was recommended. However, due to financial difficulties, the patient declined this treatment. According to the disease pathology and immunohistochemical results, endocrine therapy was suggested as an alternative. As the existing clinical trial "Pyrotinib in Combination with Letrozole in Patients with HER2-Positive, ER-Positive Metastatic Breast Cancer" (NCT04407988) was underway and the patient met the inclusion criteria, she decided to enroll in the clinical trial and apply for free treatment of pyrotinib plus letrozole.

After proper informed consent, the patient commenced continuous oral treatment of pyrotinib $400 \mathrm{mg} \mathrm{qd}+$ letrozole $2.5 \mathrm{mg}$ qd on August 27, 2020. On October 23, 2020, the patient's re-examination CT revealed that both the left axillary lymph nodes and lung nodules were significantly controlled (Figure 1C,1D). In addition, the curative effect has now been evaluated as CR, and the patient's condition has been effectively controlled (Figures $1 E, 1 F, 2$ ). During this period, no serious adverse events occurred. The only adverse reaction was mild diarrhea with increased mild glycated serum protein, but this resolved spontaneously without intervention. The patient now has a good quality of life.

All procedures performed in studies involving human participants were in accordance with the ethical standards of the institutional and/or national research committee(s) and with the Helsinki Declaration (as revised in 2013). Written informed consent was obtained from the patient for publication of this case report and accompanying images. A copy of the written consent is available for review by the editorial office of this journal.

\section{Discussion}

Approximately $15-20 \%$ of breast cancer patients are HER2positive, and $30-40 \%$ of HER2-positive early breast cancer patients develop advanced breast cancer (8). In addition, each year approximately $3-10 \%$ of breast cancer patients have distant metastasis at diagnosis. Currently, HER2+/ $\mathrm{HR}+$ breast cancer accounts for $50 \%$ of all breast cancer patients. Anti-HER2 therapy combined with chemotherapy is the recommended choice both in the NCCN and Chinese Society of Clinical Oncology (CSCO) guidelines for HER2+/HR+ breast cancer (9). However, for some HR+/ HER2+ patients who are unable to tolerate chemotherapy or meet the economic burden of the recommended therapy, 

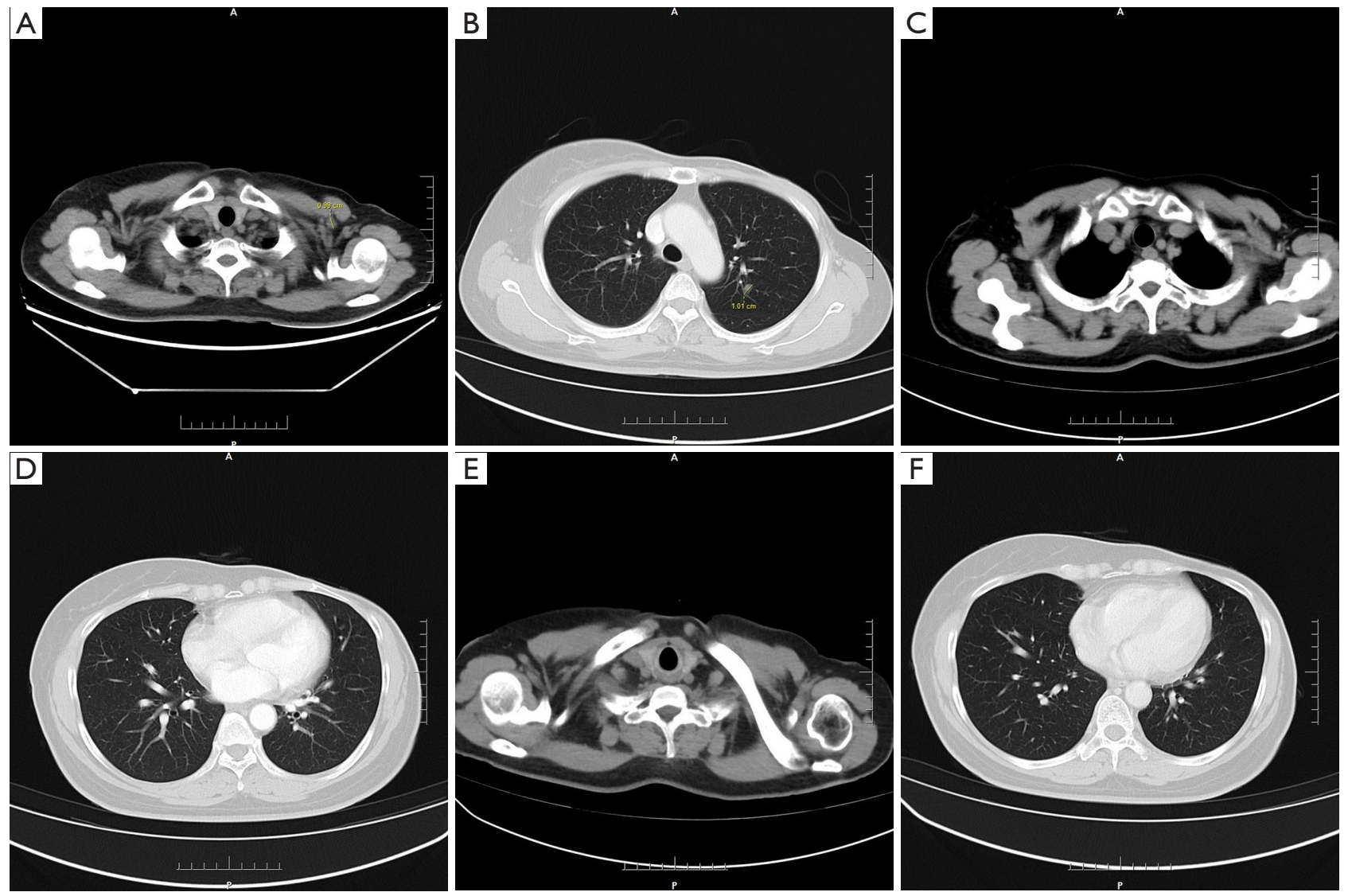

Figure 1 Computed tomography (CT) scans. (A,B) CT in August, 2020: enlarged lymph nodes in the left axillary, multiple small nodules in the lungs. (C,D) CT on October 22, 2020: complete response to combination combined therapy of pyrotinib plus letrozole therapy. (E,F) CT on March 31, 2021: 19 months after starting combination combined therapy of pyrotinib plus letrozole therapy.

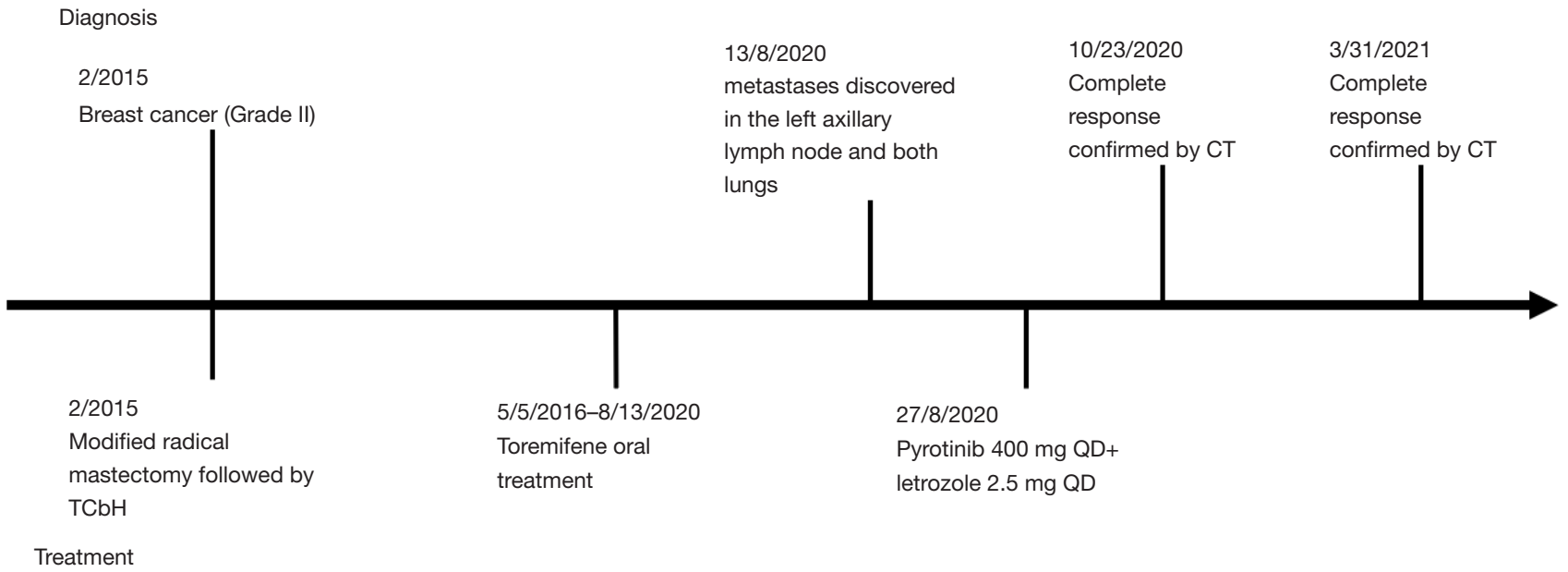

Figure 2 Timeline of diagnosis and treatment. 
anti-HER2 therapy combined with endocrine therapy is an alternative option to control their condition.

In 2015, our patient underwent extended resection of the left breast lump plus modified radical mastectomy for left breast cancer and received $\mathrm{TCbH}$ adjuvant therapy for 6 cycles. The patient was discharged after stable disease control was achieved. After discharge, she received toremifene endocrine therapy for 4 years, during which her condition was stable. Then, at a regular follow-up examination, she was diagnosed with recurrent left breast cancer with metastases in the left axillary lymph nodes and both lungs. Commonly, at the time of receiving pyrotinib with letrozole, the tumor burden of patients is extensive and the prognosis of such patients is usually poor (10). Our patient's primary lesions were ER $(3+, 60 \%)$, PR (3+, 70\%), HER2 (3+), and Ki-67 (50\%), suggesting that the cancer would be sensitive to anti-HER2 therapy and endocrine therapy. According to the current domestic and foreign guidelines, anti-HER-2 therapy combined with chemotherapy should be considered as the first-line treatment for HER2-positive and HR-positive recurrent metastatic degeneration. However, some patients who are not suitable for chemotherapy or resistant to chemotherapy agents can consider combining anti-HER2 therapy with endocrine therapy. Therefore, after screening, our patient was included in the Phase II clinical study (NCT04407988) conducted by our hospital. Since recruitment, the patient has been treated with pyrotinib combined with letrozole. Pyrotinib mainly used in the treatment of patients with HER2-positive breast cancer. Though previous studies demonstrated pyrotinib can be used for triple-negative breast cancer, it did not show a favorable effect (11). Due to widespread crosstalk between HER2 and ER signaling pathways, HER2-positive/HR-positive breast cancer is associated with resistance to anti-HER2 therapies (12). Letrozole is a highly potent and selective inhibitor of the aromatase enzyme, which could prevent the growthstimulatory activity of oestrogen and inhibit the ER signal pathway in breast cancer (6). Therefore, when combined with HER2 inhibitors, more effective anti-tumor activity may achieve in HER2-positive/HR-positive breast cancer patients.

During treatment, the patient experienced mild diarrhea, which disappeared spontaneously without intervention. Compared with the baseline period, the lymph nodes under the left armpit and the two lung nodules were reduced, and finally complete remission was achieved.

A previous TAnDEM study showed that trastuzumab combined with anastrozole in the first-line treatment of HER2+/ER+ MBC patients can prolong PFS, with a median PFS of up to 4.8 months, but no patients achieved CR (13); an EGF30008 study confirmed that TKI combined with AI had a good effect in the first-line treatment of HER2positive and HR-positive metastatic with a median PFS of 8.2 months, but only 5 (5\%) of the 111 enrolled patients achieved CR (14); an ALTERNATIVE study reported that lapatinib combined with letrozole in the treatment of HER2+/ER+ MBC patients significantly improved PFS, with a median PFS of 8.3 months, but only $7 \%$ of patients achieved CR (15). Additionally, eLEcTRA and PERTAIN studies indicated that less than $10 \%$ of HER $2+/ E R+$ MBC patients obtained CR when trastuzumab combined with letrozole was used as a first-line treatment $(16,17)$.

In this case, our patient underwent enlarged left breast lumpectomy and modified radical mastectomy for left breast cancer and then received $\mathrm{TCbH}$ adjuvant therapy for 6 cycles. After relapse, she received pyrotinib combined with letrozole as a first-line therapy and demonstrated good sensitivity to this treatment. Compared with the previous stage, the left axillary metastasis and both lung metastases were remarkably reduced and reached CR. To date, the patient's PFS is up to 19 months, the only adverse event has been mild diarrhea, and the patient's quality of life is greatly improved. Clinical trial indicated that pyrotinib plus capecitabine have prolonged PFS compared with that in lapatinib plus capecitabine group for the treatment of patients HER2-positive/HR-positive breast cancer, however, it also related with worse adverse events (18). Therefore, performance statues should be assessed before pyrotinib based-regimens conducted in HER2 positive breast cancer patients. Though the clinical benefit of antiHER2 agents combined with endocrine therapy in HER2+/ $\mathrm{HR}+\mathrm{MBC}$ patients has been acknowledged, there are still many difficulties to overcome with this treatment. These include the possibility of significant adverse reactions and the higher cost of treatment, requiring clinicians and patients to carefully weigh up the options together. In this case report, our patient with metastatic breast cancer treated by pyrotinib combined with letrozole reached CR after 2 months, and the PFS is currently 19 months. The adverse events she experienced were mild and tolerable. Thus, the first-line treatment of pyrotinib combined with letrozole may be a promising treatment option for triplepositive breast cancer patients. However, as this is a single case study report, more results from the ongoing clinical trial are needed to verify this conclusion. 


\section{Acknowledgments}

Funding: Hunan Health Commission Program (B2019089, Ning Xie). Hunan Nature and Science Foundation (2020JJ8064, Ning Xie).

\section{Footnote}

Reporting Checklist: The authors have completed the CARE reporting checklist. Available at https://dx.doi. org/10.21037/atm-21-3978

Conflicts of Interest: All authors have completed the ICMJE uniform disclosure form (available at https://dx.doi. org/10.21037/atm-21-3978). The authors have no conflicts of interest to declare.

Ethical Statement: The authors are accountable for all aspects of the work in ensuring that questions related to the accuracy or integrity of any part of the work are appropriately investigated and resolved. All procedures performed in studies involving human participants were in accordance with the ethical standards of the institutional and/or national research committee(s) and with the Helsinki Declaration (as revised in 2013). Written informed consent was obtained from the patient for publication of this case report and accompanying images. A copy of the written consent is available for review by the editorial office of this journal.

Open Access Statement: This is an Open Access article distributed in accordance with the Creative Commons Attribution-NonCommercial-NoDerivs 4.0 International License (CC BY-NC-ND 4.0), which permits the noncommercial replication and distribution of the article with the strict proviso that no changes or edits are made and the original work is properly cited (including links to both the formal publication through the relevant DOI and the license). See: https://creativecommons.org/licenses/by-nc-nd/4.0/.

\section{References}

1. Bray F, Ferlay J, Soerjomataram I, et al. Global cancer statistics 2018: GLOBOCAN estimates of incidence and mortality worldwide for 36 cancers in 185 countries. CA Cancer J Clin 2018;68:394-424.

2. $\mathrm{Xu} \mathrm{B}, \mathrm{Hu} \mathrm{X}$, Zheng $\mathrm{H}$, et al. Outcomes of re-treatment with first-line trastuzumab plus a taxane in HER2 positive metastatic breast cancer patients after (neo)adjuvant trastuzumab: A prospective multicenter study. Oncotarget 2016;7:50643-55.

3. Jemal A, Bray F, Center MM, et al. Global cancer statistics. CA Cancer J Clin 2011;61:69-90.

4. Goetz MP, Gradishar WJ, Anderson BO, et al. NCCN Guidelines Insights: Breast Cancer, Version 3.2018. J Natl Compr Canc Netw 2019;17:118-26.

5. Blair HA. Pyrotinib: First Global Approval. Drugs 2018;78:1751-5.

6. Mouridsen HT, Bhatnagar AS. Letrozole in the treatment of breast cancer. Expert Opin Pharmacother 2005;6:1389-99.

7. Wang C, Lin Y, Zhou Y, et al. Pyrotinib with trastuzumab and aromatase inhibitors as first-line treatment for HER2 positive and hormone receptor positive metastatic or locally advanced breast cancer: study protocol of a randomized controlled trial. BMC Cancer 2020;20:653.

8. Cameron D, Piccart-Gebhart MJ, Gelber RD, et al. 11 years' follow-up of trastuzumab after adjuvant chemotherapy in HER2-positive early breast cancer: final analysis of the HERceptin Adjuvant (HERA) trial. Lancet 2017;389:1195-205.

9. Cancer Professional Committee of Chinese Anti-Cancer Association. Chinese Anti-Cancer Association Guidelines and Standards for the Diagnosis and Treatment of Breast Cancer (2017 Edition). Chin J Cancer 2017;27:727-8.

10. Mederios B, Allan A, et al. Moleculer Mechanisms of Breast Cancer Metastasis to the Lung: Clinical and Experimental Perspectives. Int J Mol Sci 2019;20:2272.

11. Jiang YZ, Liu Y, Xiao Y, et al. Molecular subtyping and genomic profiling expand precision medicine in refractory metastatic triple-negative breast cancer: the FUTURE trial. Cell Res 2021;31:178-86.

12. Wang YC, Morrison G, Gillihan R, et al. Different mechanisms for resistance to trastuzumab versus lapatinib in HER2-positive breast cancers--role of estrogen receptor and HER2 reactivation. Breast Cancer Res 2011;13:R121.

13. Kaufman B, Mackey JR, Clemens MR, et al. Trastuzumab plus anastrozole versus anastrozole alone for the treatment of postmenopausal women with human epidermal growth factor receptor 2-positive, hormone receptor-positive metastatic breast cancer: results from the randomized phase III TAnDEM study. J Clin Oncol 2009;27:5529-37.

14. Johnston S, Pippen J Jr, Pivot X, et al. Lapatinib combined with letrozole versus letrozole and placebo as first-line therapy for postmenopausal hormone receptor-positive metastatic breast cancer. J Clin Oncol 2009;27:5538-46. 
15. Johnston SRD, Hegg R, Im SA, et al. Phase III, Randomized Study of Dual Human Epidermal Growth Factor Receptor 2 (HER2) Blockade With Lapatinib Plus Trastuzumab in Combination With an Aromatase Inhibitor in Postmenopausal Women With HER2Positive, Hormone Receptor-Positive Metastatic Breast Cancer: Updated Results of ALTERNATIVE. J Clin Oncol 2021;39:79-89.

16. Huober J, Fasching PA, Barsoum M, et al. Higher efficacy of letrozole in combination with trastuzumab compared to letrozole monotherapy as first-line treatment in patients with HER2-positive, hormone-receptor-positive metastatic breast cancer - results of the eLEcTRA trial.

Breast 2012;21:27-33.

Cite this article as: Xie N, Liu L, Tian C, Hu Z, Ouyang Q. Complete response to pyrotinib combined with letrozole as first-line treatment of HER2-positive/HR-positive breast cancer: a case report. Ann Transl Med 2021;9(16):1356. doi: 10.21037/atm-21-3978
17. Rimawi M, Ferrero JM, de la Haba-Rodriguez J, et al. First-Line Trastuzumab Plus an Aromatase Inhibitor, With or Without Pertuzumab, in Human Epidermal Growth Factor Receptor 2-Positive and Hormone ReceptorPositive Metastatic or Locally Advanced Breast Cancer (PERTAIN): A Randomized, Open-Label Phase II Trial. J Clin Oncol 2018;36:2826-35.

18. Ma F, Ouyang Q, Li W, et al. Pyrotinib or Lapatinib Combined With Capecitabine in HER2-Positive Metastatic Breast Cancer With Prior Taxanes, Anthracyclines, and/or Trastuzumab: A Randomized, Phase II Study. J Clin Oncol 2019;37:2610-9.

(English Language Editor: D Fitgerlad) 